\title{
Improving telestroke treatment times through a quality improvement initiative in a Singapore emergency department
}

Rupeng Mong ${ }^{1}$, MBBS, MRCEM, Ling $\underline{T i a h}^{1}$, MBBS, MPH, Michelle $\underline{\text { Wong }}{ }^{1}$, MBBS, Camlyn $\underline{\operatorname{Tan}}^{1}$, MBBS, MRCS

INTRODUCTION Telestroke allows for remote determination of suitability for treatment with thrombolysis in patients with acute ischaemic stroke. However, this approach is time-dependent and most centres have yet to achieve the recommended treatment times. We describe a quality improvement initiative aimed at improving the telestroke workflow and treatment times at our centre.

METHODS A multidisciplinary workgroup comprising clinicians, stroke case managers and radiology staff was formed to oversee the initiative. A phase-by-phase review of the existing workflow was done to identify the reasons for delay. Phase-specific measures were then introduced to address these delays, and a data-monitoring system was established to track the impact of these measures. The initiatives were implemented through four Plan-Do-Study-Act cycles. The door-to-needle (DTN) times for thrombolysis and clinical outcomes before and after the interventions were compared. RESULTS A total of 104 patients were evaluated. The median DTN time improved from 96 minutes to 78 minutes post implementation of initiatives $(p=0.003)$. Fewer patients had symptomatic intracranial haemorrhages $(8.5 \%$ vs. $24.2 \%$; $p=0.03)$, and more patients had improvements in their National Institutes of Health Stroke Scale score $(47.9 \%$ vs. $25.0 \%$; $\mathrm{p}=0.031$ ) after the initiatives were introduced.

CONCLUSION The quality improvement initiative resulted in a reduction in median DTN time. Our approach allowed for a systematic method to resolve delays within the telestroke workflow. This initiative is part of an ongoing effort aimed at providing thrombolysis safely to eligible patients in the shortest possible time.

Keywords: door-to-needle time, quality improvement, stroke, telemedicine

\section{INTRODUCTION}

The use of tissue plasminogen activator (tPA) for thrombolysis in acute ischaemic stroke improves neurological outcomes. ${ }^{(1)}$ However, its benefit is time-dependent, with delayed administration leading to an increased risk of intracranial haemorrhage. ${ }^{(2,3)}$ Patients with acute ischaemic stroke without contraindications for IPA should have their evaluation completed and TPA initiated within 60 minutes. ${ }^{(4,5)}$ Unfortunately, evidence shows that many patients do not receive tPA in a timely manner. ${ }^{(6-9)}$

Changi General Hospital (CGH), a 1,000-bed acute regional hospital in the eastern part of Singapore, sees approximately 150,000 emergency department (ED) attendances annually. As there is no on-site neurology support, telestroke was implemented in 2010 to facilitate the assessment of stroke patients by a neurologist from a remote location. ${ }^{(10)}$ The telestroke workflow has been under much scrutiny, as the additional processes that are required, as compared with conventional face-to-face assessment by a neurologist, may lead to delays in thrombolysis. ${ }^{(11)}$ An earlier audit of patients receiving tPA at our centre in 2011 found the median door-to-needle (DTN) time to be 92.7 minutes. ${ }^{(12)}$ As part of the hospital's efforts to improve stroke care, a workgroup was formed in 2012 to monitor and improve the telestroke service.

The primary objective of this study was to describe the quality improvement initiatives undertaken and their impact on DTN time for patients receiving thrombolysis. The secondary objective was to analyse changes to clinical indicators, including improvements to the National Institutes of Health Stroke Scale (NIHSS) score after thrombolysis, rate of intracranial haemorrhage and inpatient mortality, following the introduction of these initiatives.

\section{METHODS}

$\mathrm{CGH}$ runs a 24-hour telestroke service that involves the collaborative efforts of medical staff from the departments of emergency medicine, radiology and neurology, as well as stroke case managers. It is based on the hub-and-spoke model, where eligible patients presenting to our centre (i.e. spoke) with acute ischaemic stroke are evaluated by off-site neurologists from the National Neuroscience Institute (NNI), Singapore (i.e. hub). The web-based telemedicine process is supported by the Krixi Care ${ }^{\mathrm{TM}}$ program, allowing for real-time audio-visual link, including image transfer for remote viewing of computed tomography (CT) images.

A telestroke workgroup, comprising clinicians, stroke case managers and radiology personnel from the two centres (i.e. $\mathrm{CGH}$ and $\mathrm{NNI}$ ), was formed to oversee this quality improvement initiative. The adopted approach involved a phase-by-phase review of the existing telestroke workflow, identifying potential areas for improvement, followed by the application of relevant change concepts for testing. These were achieved through four Plan-Do-Study-Act (PDSA) cycles. 


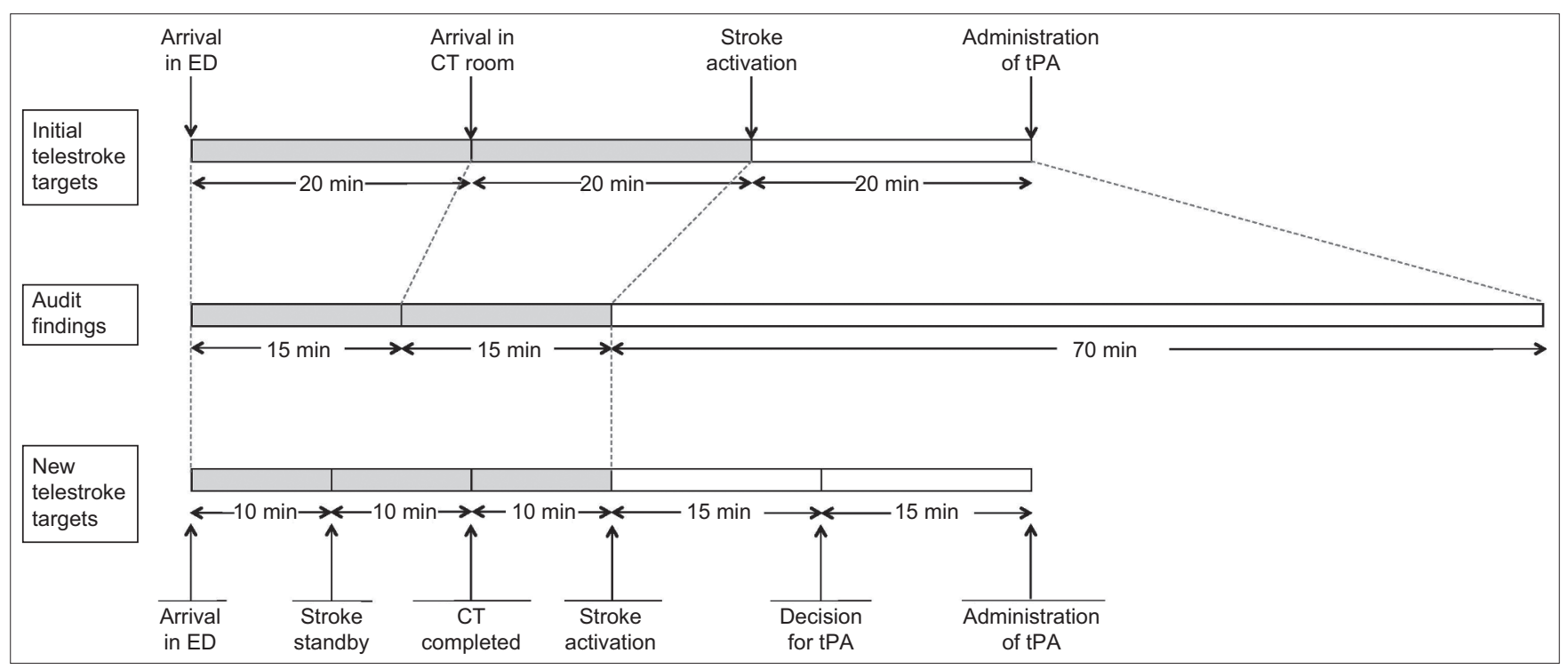

Fig. 1 Diagram shows initial target timings and audit findings, and the new target timings proposed by the workgroup. CT: computed tomography; ED: emergency department; tPA: tissue plasminogen activator

The objective of the first PDSA cycle was to review and redefine the target times for the various phases of telestroke. Prior to this, the process of telestroke was divided into three phases: patient arrival in ED to arrival in $\mathrm{CT}$ room; arrival in $\mathrm{CT}$ room to stroke activation; and stroke activation to administration of tPA. The target time for each phase was 20 minutes. Through a series of audits, the third phase of telestroke, which included setup of video-conferencing equipment, assessment by the neurologist, obtaining of informed consent and preparation of tPA, was found to require markedly more time than allocated. With this knowledge, the phases of telestroke were redefined into five phases to allow for more precise monitoring, with more time allocated to the later phases. The five phases were: (1) arrival in ED to stroke standby; (2) stroke standby to completion of CT imaging; (3) completion of CT imaging to stroke activation; (4) stroke activation to decision for IPA; and (5) decision for tPA to administration of tPA. Fig. 1 compares the time allocation for the initial telestroke phases with the findings from the audit, and shows the time allocation for the redefined telestroke phases.

The aim of the second PDSA cycle was to establish a reliable data-monitoring and feedback system to track the impact of the initiatives. Details on timings for the various phases of telestroke and feedback gathered from medical staff involved in the telestroke were collected and analysed regularly. The stroke case manager was in charge of collating this data. New initiatives in this cycle included: (a) use of standardised data collection forms; (b) time stamping for the start and end of each phase for accurate tracking of timings; (c) monthly analysis of data; and (d) creation of a channel for ad-hoc feedback from ground staff.

The subsequent PDSA cycles focused on the implementation, monitoring and review of phase-specific initiatives. The key events for the first three phases included triage, initial physician assessment and transfer of patient for CT imaging. To expedite the identification of eligible patients, all cases that were pre-notified by emergency medical services and conveyed by ambulances had their initial assessment done by an ED physician in the critical care area. Patients who were not conveyed by ambulances and triaged in the ambulatory area were screened for features of stroke using the Face-Arm-Speech-Test assessment tool. ${ }^{(13)}$ Indications and contraindications to thrombolysis were listed in the stroke clinical care pathway, which was easily accessible for reference. When a patient was deemed a potential candidate for thrombolysis, a 'stroke standby' would be initiated to the call centre. This triggered the CT room to prepare to receive the patient and alert the radiologist to stand by for interpretation of images. The neurologist at NNI and on-site stroke case manager would also be informed to stand by for a possible telestroke consultation. Once the CT images were reviewed and no contraindications to thrombolysis were found, the radiologist (registrar/senior resident grade and above) would call for 'stroke activation'. This automatically prompted the call centre to activate all relevant stroke team members.

Applying the principles of parallel processing, the key events within Phase 4 (assessment via video-conferencing and decision by neurologist) and Phase 5 (obtaining consent and administration of tPA) were reviewed to determine which tasks could be brought forward and done concurrently in the earlier phases. Video-conferencing was held through a mobile computer cart that was brought to the patient's bedside, and log-in access to Krixi Care was required. Previously, video-conferencing was prepared only after CT imaging and confirmation that the patient had no contraindications to proceed. As part of the new initiative, the equipment was prepared earlier, usually while the patient was being sent for CT imaging. This also allowed time for the backup video-camera system to be activated should the main system malfunction. Treatment with IPA was explained to the patient by the ED physician with the aid of a patient information sheet. This was usually done after a decision for thrombolysis had been made by the neurologist. To expedite this process, the ED physician would explain the potential use of thrombolysis 


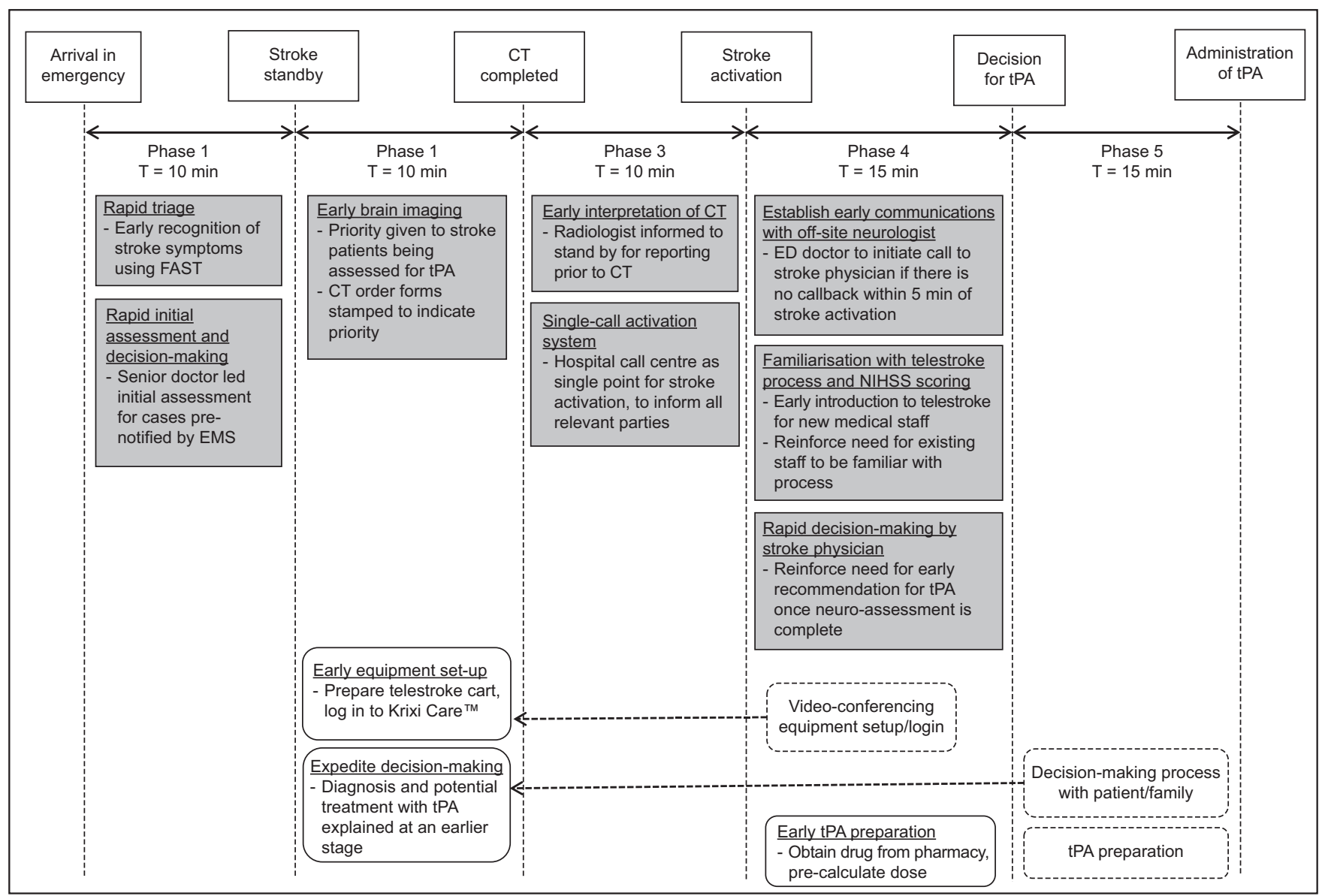

Fig. 2 Summary of quality improvement initiatives. CT: computed tomography; ED: emergency department; EMS: emergency medical service; FAST: facearm-speech-test; NIHSS: National Institutes of Health Stroke Scale; NNI: National Neuroscience Institute; tPA: tissue plasminogen activator

before a definite decision was made. After the neurologist had completed the telestroke consultation, any additional patientspecific information or risks (not previously explained or found in the patient information sheet) would then be communicated to the patient before the actual consent was taken. In situations where the patient was unable to give informed consent, the decision to proceed with thrombolysis would be jointly made by the ED physician and neurologist. Drug preparation (obtaining tPA from the pharmacy and determining the dose using a weightbased, pre-calculated drug table) was also done earlier upon stroke activation.

In addition, initiatives were introduced to minimise delays in communication with the off-site neurologists. The neurologist at $\mathrm{NNI}$ was directly informed of the stroke activation through the call centre. Upon receiving the notification of stroke activation, the off-site neurologist would initiate a call-back to the ED and start the assessment of the patient via video-conferencing. During the audit, there were situations where the call-back was delayed. To establish timely communication with the off-site neurologist, the treating doctor would call the neurologist if there was no call-back within five minutes of stroke activation.

As the process of telestroke involved multiple tasks (including early identification of stroke features, coordination with radiology and neurology departments, preparation of telestroke equipment, assessment of NIHSS score, obtaining of consent and administration of tPA), there was a learning curve to the entire process, especially for physicians and nurses who were new to the ED. Due to a lack of familiarity with the routine or awareness about the importance of timeliness in the management of these patients, a session about telestroke, including a demonstration of the Krixi Care telestroke program, was incorporated into the regular departmental teaching curriculum. Fig. 2 summarises the key initiatives carried out through the five phases.

This observational study comparing the median DTN time for patients receiving tPA before and after the introduction of the quality improvement initiative was approved by the institutional review board. The study period was from 9 April 2012 to 31 December 2013, and included all patients aged $\geq 21$ years who presented with acute ischaemic stroke and were treated with IPA in the ED. The cut-off time for IPA administration was 4.5 hours from the time of onset of stroke symptoms. Patients presenting with atypical features and inconsistent history and those who developed features of stroke only after arrival in the ED were excluded. Trained research assistants extracted data from the hospital stroke database and patient medical records.

The following parameters were extracted: patient demographics; past medical history; DTN time; time taken for each phase of telestroke; NIHSS score on arrival and at 24 hours post thrombolysis; and inpatient mortality. Data collected was analysed and compared among patients treated before (9 April 2012 to 28 February 2013; pre-intervention group) and after (1 March 2013 to 31 December 2013; post-intervention group) 
the initiatives. The primary outcome measure was median DTN time. Secondary outcome measures included: frequency of intracranial haemorrhage post thrombolysis; improvement in NIHSS score from baseline at 24 hours post thrombolysis (defined as a decrease in NIHSS score $\geq 4$ ); and inpatient mortality rate. Data analysis was done using SPSS Statistics version 19.0 (IBM Corp, Armonk, NY). Mann-Whitney $U$ test was used to analyse the primary outcome for statistical significance, which was defined

Table I. Demographics and clinical characteristics of patients receiving thrombolysis before and after the implementation of quality improvement initiative.

\begin{tabular}{|c|c|c|}
\hline \multirow[t]{2}{*}{ Parameter } & \multicolumn{2}{|c|}{ Median (IQR)/\% } \\
\hline & $\begin{array}{l}\text { Pre-intervention } \\
\text { group }(n=33)\end{array}$ & $\begin{array}{l}\text { Post-intervention } \\
\text { group }(n=71)\end{array}$ \\
\hline Age (yr) & $66(36-90)$ & $66(30-90)$ \\
\hline Male gender & 66.7 & 64.8 \\
\hline \multicolumn{3}{|l|}{ Race } \\
\hline Chinese & 54.5 & 76.1 \\
\hline Malay & 21.2 & 8.5 \\
\hline Indian & 15.2 & 9.9 \\
\hline Others & 9.1 & 5.6 \\
\hline Smoker & 36.4 & 33.8 \\
\hline \multicolumn{3}{|l|}{ Past medical history } \\
\hline Diabetes mellitus & 48.5 & 42.3 \\
\hline Hypertension & 69.7 & 69.0 \\
\hline Dyslipidaemia & 75.8 & 73.2 \\
\hline Atrial fibrillation & 33.3 & 28.2 \\
\hline Ischaemic heart disease & 51.5 & 33.8 \\
\hline Peripheral vascular disease & 0.0 & 4.2 \\
\hline Previous CVA/TIA & 15.2 & 16.9 \\
\hline Carotid artery stenosis & 24.2 & 22.5 \\
\hline \multicolumn{3}{|l|}{ Clinical characteristics } \\
\hline Onset-to-arrival (min) & $66(45-97)$ & $70(45-109)$ \\
\hline Initial NIHSS score & $12(7-15)$ & $10(7-17)$ \\
\hline
\end{tabular}

CVA: cerebrovascular accident; IQR: interquartile range; NIHSS: National Institutes of Health Stroke Scale; TIA: transient ischaemic attack as a p-value $<0.05$. Chi-square test was used to analyse for statistical significance in clinical outcomes between the pre- and post-intervention groups.

\section{RESULTS}

A total of 104 patients with acute ischaemic stroke were treated with tPA during the study period. Table I shows the patient demographics and baseline clinical characteristics. The median age and gender distribution were comparable between groups, but the percentage of ethnic Chinese was lower in the preintervention group.

Table II shows the DTN times and clinical outcomes of patients before and after intervention. The median DTN time for tPA administration was 96 (interquartile range [IQR] 84-110) minutes for the pre-intervention group, and decreased to 78 (IQR 68-101) minutes for the post-intervention group $(p=0.003)$. The timings for each of the five phases were analysed and compared between the groups. The median time for Phase 2 improved from 8 (IQR 5-12) minutes before the initiative to 3 (IQR 2-5) minutes after the initiative $(p<0.001)$, while the median time for Phase 4 improved from 35 (IQR 28-42) minutes to 26 (IQR 20-32) minutes $(p<0.001)$. There were no significant changes in the median times for Phases 1, 3 and 5. Fig. 3 compares the timings of each phase using box and whisker plots.

There were significantly fewer patients with symptomatic intracranial haemorrhages post thrombolysis in the postintervention group $(8.5 \%$ vs. $24.2 \%$; $p=0.030)$. There were also more patients with improvements in NIHSS score at 24 hours post thrombolysis in the post-intervention group $(47.9 \%$ vs. $25.0 \%$; $p=0.031)$. No significant difference was noted in inpatient mortality between the pre- and post-intervention groups $(6.1 \%$ $7.0 \% ; p=1.000)$.

\section{DISCUSSION}

Our results showed that the new measures introduced helped to reduce delays in DTN time. Although the initiatives did not achieve a DTN time of less than 60 minutes, there was a

Table II. Door-to-needle (DTN) times and clinical outcomes in the pre-intervention and post-intervention groups.

\begin{tabular}{|lll|}
\hline \multirow{2}{*}{ Outcomes } & \multicolumn{1}{c|}{ Median (IQR)/\% } & p-value \\
\cline { 2 - 3 } & Pre-intervention group $(\mathbf{n}=\mathbf{3 3})$ & Post-intervention group (n= 71) \\
\hline DTN (min) & $96(84-110)$ & $78(68-101)$ \\
\hline NIHSS score & 32 & 67 \\
\hline No. of patients analysed* & 25.0 & 47.9 \\
\hline Improvement in NIHSS score at 24 hr & & \\
\hline ICH & 33 & 70 \\
\hline No. of patients analysed & & \\
\hline No ICH & 48.5 & 72.9 \\
\hline Symptomatic ICH & 24.2 & 8.5 \\
\hline Inpatient mortality & & \\
\hline No. of patients analysed & 33 & 71 \\
\hline Inpatient mortality & 6.1 & 7.0 \\
\hline
\end{tabular}

*NIHSS scores at 24 hours were not available for one patient from the pre-intervention group and four patients from the post-intervention group. + Follow-up imaging was not available for one patient from the post-intervention group. ICH: intracranial haemorrhage; IQR: interquartile range; NIHSS: National Institutes of Health Stroke Scale 


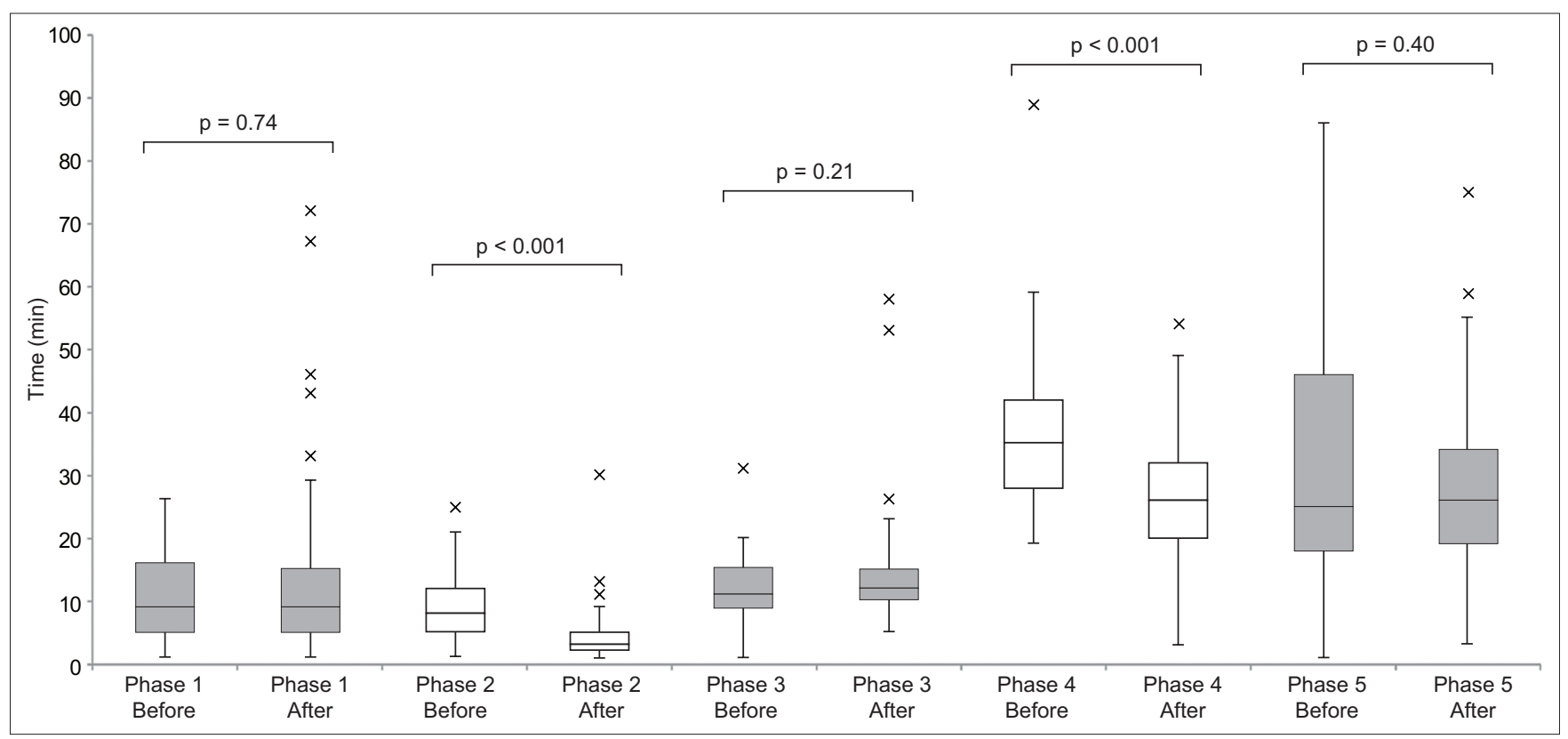

Fig. 3 Box and whisker plot shows the time taken for each phase of telestroke before and after the initiatives.

significant improvement from what was previously achieved. Other centres offering telestroke have reported comparable DTN times of $61-106$ minutes. ${ }^{(8,11,14-16)}$ The wide range of timings in these studies suggests that multiple factors can affect DTN times, including differences in workflow among centres, organisation of EDs, available resources, as well as training and experience of medical staff.

The American Heart Association/American Stroke Association's Target: Stroke initiative provided ten key strategies to achieve timely tPA administration, ${ }^{(4)}$ including: pre-notification by emergency medical service; use of rapid triage protocols and stroke tools; early stroke team notification; early acquisition and interpretation of brain imaging; rapid laboratory testing; rapid access to and early preparation of tPA; a team-based approach; and prompt data feedback. Other strategies have also been described, including locating the $\mathrm{CT}$ room within the ED, direct transfer of patients onto the CT table upon arrival and use of point-of-care testing. ${ }^{(17-19)}$ The workgroup studied several of these measures that were not already in place and implemented those that were feasible in our setting. Telestroke, however, presents additional challenges compared with conventional face-to-face assessment by a neurologist. Additional time is required for equipment setup and clinical evaluation, and communication difficulties and equipment failure could lead to further delays. Our initiatives, which also addressed these issues, focused on the need to prepare telestroke video-conferencing equipment and set up the web-based program in advance, ensuring that our doctors were familiar with the telestroke process and reinforcing the need for rapid decision-making by the off-site neurologists.

Our approach was to divide the telestroke process into five phases. A detailed analysis of each phase allowed us to focus on areas that caused the most delays and to introduce initiatives to address these phase-specific delays. Our system of data monitoring, including phase-by-phase monitoring of telestroke timings, enabled us to monitor the impact of the various initiatives introduced, which would not otherwise be apparent if only the DTN time was monitored.

Our median door-to-imaging time (i.e. time of ED arrival to completion of brain $\mathrm{CT}$ ) improved from 17 minutes to 13 minutes. This was predominantly due to earlier access to brain imaging. The main initiative introduced during this phase gave patients being assessed for thrombolysis priority to $\mathrm{CT}$ imaging. Our median time was within the 25 minutes recommended by the Target: Stroke initiative. ${ }^{(4)}$ This result showed that we could potentially reduce our target time, to allow more time to be allocated to the later phases, which we found required more time than was previously anticipated.

The median time for video-conferencing and decision by the off-site neurologist (Phase 4) showed an improvement from 35 minutes to 26 minutes. This was, however, still above our target of 15 minutes. Based on feedback received, there were situations where, despite attempts at early setup, equipment failure or connection issues still led to delays. Strengthening the support from Krixi Care vendors and our information technology team, we continue to work closely with them to minimise disruptions due to technical issues and ensure that the software is constantly updated.

Upon completion of patient assessment, a definitive recommendation by the neurologist regarding $\mathrm{PA}$ use is required to set in motion the next series of tasks. A delay in the decisionmaking process would contribute to an increase in DTN time. To facilitate this, the attending ED physician should proactively provide the neurologist with pertinent clinical information that is not available during video-conferencing. At the same time, it would be ideal for the neurologist, who has to make the final decision about IPA, to receive the information first-hand from the attending ED physician.

In the present study, not all phases showed improvement in timing. The median time for Phase 5 was 26 minutes, similar to that before the initiatives were implemented and longer than our 
target time of 15 minutes. The delay was largely due to the time required for obtaining consent for IPA administration. For patients to make an informed decision, the risks and benefits of tPA must be explained thoroughly, and this can be time-consuming. Although we proposed to start this process earlier, it was not always feasible to do so concurrently with other competing tasks, such as setting up of equipment and video-conferencing with the neurologist. Moreover, the patient may be away in the CT room. Moving forward, the workgroup will explore other initiatives that could potentially expedite this process. New decision support tools consisting of simple diagrams that illustrate the risks and benefits of tPA have been introduced. Reviewing other institutions' process of obtaining consent for tPA may also provide insight to how we can further streamline this process.

Improved DTN time has been shown to result in better clinical outcomes, including reductions in symptomatic intracranial haemorrhage, decreased inpatient mortality rates and improved functional outcomes..$^{(1,4,20)}$ In our study, there were fewer cases of symptomatic intracranial haemorrhage in the post-intervention group. We also looked at the difference in NIHSS scores on arrival and at 24 hours post thrombolysis as a marker of early neurological improvement; ${ }^{(21)}$ we found a higher percentage of patients with improved scores in the post-intervention group. However, as this was a non-randomised observational study, these differences in clinical outcomes may have been due to other factors, including differences in patient characteristics between groups. We were unable to demonstrate a difference in inpatient mortality rates. This may have been due to our relatively small sample size, which was not powered to detect any differences.

This study has several limitations. Firstly, it was possible that improvements in DTN times were influenced by other factors. For instance, medical staff could have become increasingly familiar with the process through the study period, but the extent to which this has affected the DTN time is uncertain. Secondly, data regarding the frequency of software or equipment failure resulting in delays was not available, and these incidents were not always documented in the treating physician's note. Thus, we were unable to determine the extent to which this has contributed to delays. Finally, as our primary focus was the impact of initiatives on DTN times, certain clinical outcomes, such as functional outcomes and discharge destinations, were not tracked. Moreover, sample size was not pre-calculated to detect potential differences in mortality rates.

In conclusion, our quality improvement initiative resulted in an overall reduction in the median DTN time for patients with acute ischaemic stroke receiving thrombolysis. While we did not achieve the target median DTN time of 60 minutes during the study period, the measures instituted and lessons learnt as part of the PDSA cycles have helped to establish a mechanism for ongoing improvement efforts. Anchored by our multidisciplinary team with close collaboration between our centre and NNI, we strive to continually improve our services with the aim of providing thrombolysis safely to eligible patients in the shortest time possible.

\section{REFERENCES}

1. National Institute of Neurological Disorders and Stroke rt-PA Stroke Study Group. Tissue plasminogen activator for acute ischemic stroke. N Engl J Med 1995; 333:1581-7

2. Saver JL, Fonarow GC, Smith EE, et al. Time to treatment with intravenous tissue plasminogen activator and outcome from acute ischemic stroke. JAMA; 309:2480-8

3. Lees KR, Bluhmki E, von Kummer R, et al. Time to treatment with intravenous alteplase and outcome in stroke: an updated pooled analysis of ECASS, ATLANTIS, NINDS, and EPITHET trials. Lancet 2010; 375:1695-703.

4. Fonarow GC, Smith EE, Saver JL, et al. Improving door-to-needle times in acute ischemic stroke: the design and rationale for the American Heart Association/ American Stroke Association's Target: Stroke initiative. Stroke 2011; 42:2983-9.

5. Jauch EC, Saver JL, Adams HP Jr, et al; American Heart Association Stroke Council; Council on Cardiovascular Nursing; Council on Peripheral Vascular Disease; Council on Clinical Cardiology. Guidelines for the early management of patients with acute ischemic stroke: a guideline for healthcare professionals from the American Heart Association/American Stroke Association. Stroke 2013; 44:870-947.

6. Parra C, Jódar-Sánchez F, Jiménez-Hernández MD, et al. Development, implementation, and evaluation of a telemedicine service for the treatment of acute stroke patients: telestroke. Interact J Med Res 2012; 1:e15.

7. Demaerschalk BM, Raman R, Ernstrom K, Meyer BC. Efficacy of telemedicine for stroke: pooled analysis of the Stroke Team Remote Evaluation Using a Digital Observation Camera (STRokE DOC) and STRokE DOC Arizona telestroke trials. Telemed J E Health 2012; 18:230-7.

8. Adams RJ, Debenham E, Chalela J, et al. REACH MUSC: a telemedicine facilitated network for stroke: initial operational experience. Front Neurol 2012; 3:33.

9. Schwamm LH, Rosenthal ES, Hirshberg A, et al. Virtual TeleStroke support for the emergency department evaluation of acute stroke. Acad Emerg Med 2004; 11:1193-7

10. Singh $\mathrm{R}, \mathrm{Ng} \mathrm{WH}$, Lee KE, et al. Telemedicine in emergency neurological service provision in Singapore: using technology to overcome limitations. Telemed J E Health 2009; 15:560-5.

11. Chowdhury M, Birns J, Rudd A, Bhalla A. Telemedicine versus face-to-face evaluation in the delivery of thrombolysis for acute ischaemic stroke: a single centre experience. Postgrad Med J 2012; 88:134-7.

12. Ang SH, Tan C, Singh R. Telestroke: rapid treatment of acute ischemic stroke patients using telemedicine in a Singapore emergency department. Eur J Emerg Med 2013; 20:322-6.

13. Rudd M, Buck D, Ford GA, Price Cl. A systematic review of stroke recognition instruments in hospital and prehospital settings. Emerg Med J 2016; 33:818-22.

14. Bruno A, Lanning KM, Gross $\mathrm{H}$, et al. Timeliness of intravenous thrombolysis via telestroke in Georgia. Stroke 2013; 44:2620-2.

15. Sauser K, Levine DA, Nickles AV, Reeves MJ. Hospital variation in thrombolysis times among patients with acute ischemic stroke: the contributions of door-toimaging time and imaging-to-needle time. JAMA Neurol 2014; 71:1155-61.

16. Nardetto L, Dario C, Tonello S, et al. A one-to-one telestroke network: the first Italian study of a web-based telemedicine system for thrombolysis delivery and patient monitoring. Neurol Sci 2016; 37:725-30.

17. Lindsberg PJ, Häppölä $O$, Kallela $M$, et al. Door to thrombolysis: ER reorganization and reduced delays to acute stroke treatment. Neurology 2006; 67:334-6.

18. Meretoja A, Strbian D, Mustanoja S, et al. Reducing in-hospital delay to 20 minutes in stroke thrombolysis. Neurology 2012; 79:306-13.

19. Meretoja A, Weir L, Ugalde M, et al. Helsinki model cut stroke thrombolysis delays to 25 minutes in Melbourne in only 4 months. Neurology 2013; 81:1071-6

20. Fonarow GC, Zhao X, Smith EE, et al. Door-to-needle times for tissue plasminogen activator administration and clinical outcomes in acute ischemic stroke before and after a quality improvement initiative. JAMA 2014; 311:1632-40.

21. Kharitonova T, Mikulik R, Roine RO, et al; Safe Implementation of Thrombolysis in Stroke Investigators. Association of early National Institutes of Health Stroke Scale improvement with vessel recanalization and functional outcome after intravenous thrombolysis in ischemic stroke. Stroke 2011; 42:1638-43. 